In the meantime, if the lessons of this volume are taken to heart with those of the first report, the perinatal mortality will continue to fall and some geographical and social differentials will be reduced.

The book deserves and will have a great success.

Progress in Medical Virology, Vol. 10. Edited by J. L. MelNICK; with 20 contributors. (Pp. xviii +502; 50 figures +23 tables. sFr./DM 88.; \$21.10; 176s.) Basel and New York: S. Karger. Distribution in U.K.: Academic Press, London. 1968.

This is the 10th volume in the series on 'Progress in Medical Virology' first published in 1958. Once again J. L. Melnick, the Editor, has drawn together a panel of experts from all over the world to write about current progress in medical virology, and once again he has achieved a fine balance in his choice of subject matter in this rapidly developing branch of medicine. There is much of interest in this volume to paediatricians and virologists alike. Two chapters on 'the pathogenesis of viral infections of the fetus' and on 'congenital rubella' will be found of special interest and will bring the reader up-to-date with the latest information on the action of viruses on the mammalian fetus and on the phenomenon of virus persistence. Further chapters on 'virus interference', on 'evaluation of antiviral drugs in volunteers', and on various aspects of laboratory diagnosis provide good and interesting reading. Throughout this volume the same high standard of presentation has been maintained, and each chapter contains a comprehensive and up-to-date bibliography. This volume is a valuable addition to a valuable scientific series, and is strongly recommended to virologists and clinicians who have an interest not only in prophylaxis, diagnosis, and treatment of viral disease, but in the broader aspects of disease caused by this important group of infectious agents

\section{Endocrine and Genetic Diseases of Childhood.}

Edited by LytT J. Gardner. (Pp. xviii +1072; illustrated + tables. 227s.) Philadelphia and London: W. B. Saunders. 1969.

This thousand-page book covers almost the entire field of genetically determined and endocrine conditions in childhood, and its 57 authors are each of them worldrenowned specialists in their own discipline. It is, indeed, as claimed in the preface, a 'multi-authored text book ...' It can, however, scarcely be considered as suitable reading (as also claimed in the preface) for 'the student, the house officer and the practitioner ...' The chapters are, for the most part, up-to-date monographs or review articles on highly specialized topics: some are brilliantly successful, and could be most valuable to the paediatrician, and possibly even to the registrar intent on a consultant career. Others are too detailed and erudite for any but the specialist himself, and yet others, and they are too many and long and tiresome to read, not infrequently add confusion to subjects already difficult to understand. Moreover, there is too frequently lack of balance or proportion between one subject and another. What, for instance, whether as student, practitioner, or highly-trained specialist, is one to make on the one hand, of Chapter 9 with 111 pages devoted to chromosome disorders, including minute details of tissue culture technique and slide preparation, or Chapter 10 with 25 pages on periodic paralysis, when cystic fibrosis is dealt with in a chapter of barely $1 \frac{1}{2}$ pages in which management is covered in a paltry 20 lines and its pulmonary complications dismissed in 6 cryptic words?

It is also difficult to fathom the reasons why the only coloured plate illustrates, in coppery hue, four children with varying degrees of hypothyroidism. In other ways, the printing, binding, illustrations, and figures are of the high order one has come to expect of this publishing house. It is doubtful whether this expensive textbook will satisfy enough readers to make its purchase justifiable by, for instance, a hospital library.

\section{Calorie Deficiencies and Protein Deficiencies.} Proceedings of a Colloquium held at Cambridge, April 1967. Edited by R. A. MCCANCE and E. M. Widdowson. (Pp. xiv + 386; 129 figures. 80s.) London: J. and A. Churchill. 1968.

This book documents the proceedings of a small private colloquium held in Cambridge in April 1967. The time between the meeting and the appearance of the book is probably as short as can be attained and is not in this case serious. Nor, for the most part, have the usual deficiencies of congress reports been repeated here, even though a few strange colloquialisms have been retained in the discussions.

A galaxy of well-known talent from all parts of the clinical and experimental world of undernutrition has contributed a series of excellent, informative reviews, and a vivid picture of kwashiorkor and marasmus with their intermediate and allied conditions emerges. As might have been expected from the editors, a generous portion of the book is devoted to experimental investigations in animals, and to a thorough critical consideration of the validity of their extrapolation to man. An impressive case is once more made for the usefulness of combining animal with clinical experiments. Nevertheless, more than half the book is concerned with clinical aspects, human pathology and metabolic patterns, problems of treatment, and such topics as the roles of anaemia, vitamin deficiencies, and toxins.

One is again left wondering just how helpful can the detailed study of the results of disease in advanced human cases be to the discovery of aetiology and pathogenesis. Many of the most characteristic changes of kwashiorkor (for example in amino acid metabolism) are adaptive; and their minute description therefore contributes very little to an understanding of the important early development of the disease. Attempts (and failures) to produce animal models may well contribute more real information about the human condition than many clinicians at present recognize.

There has been a largely sterile battle in recent years over the nomenclature of the diseases considered 